Published in final edited form as:

Chem Biol Interact. 2015 June 5; 234: 38-44. doi:10.1016/j.cbi.2014.12.038.

\title{
Discovery of a series of aromatic lactones as ALDH1/2-directed inhibitors
}

\author{
Cameron D. Buchman, Krishna K. Mahalingan, and Thomas D. Hurley* \\ Department of Biochemistry and Molecular Biology, Indiana University School of Medicine, \\ Indianapolis, IN 46202-5122, United States
}

\begin{abstract}
In humans, the aldehyde dehydrogenase superfamily consists of 19 isoenzymes which mostly catalyze the $\mathrm{NAD}(\mathrm{P})^{+}$-dependent oxidation of aldehydes. Many of these isoenzymes have overlapping substrate specificities and therefore their potential physiological functions may overlap. Thus the development of new isoenyzme-selective probes would be able to better delineate the function of a single isoenyzme and its individual contribution to the metabolism of a particular substrate. This specific study was designed to find a novel modulator of ALDH2, a mitochondrial ALDH isoenzyme most well-known for its role in acetaldehyde oxidation. 53 compounds were initially identified to modulate the activity of ALDH2 by a high-throughput esterase screen from a library of 63,000 compounds. Of these initial 53 compounds, 12 were found to also modulate the oxidation of propionaldehyde by ALDH2. Single concentration measurements at $10 \mu \mathrm{M}$ compound were performed using ALDH1A1, ALDH1A2, ALDH1A3, ALDH2, ALDH1B1, ALDH3A1, ALDH4A1, and/or ALDH5A1 to determine the selectivity of these 12 compounds towards ALDH2. Four of the twelve compounds shared an aromatic lactone structure and were found to be potent inhibitors of the ALDH1/2 isoenzymes, but have no inhibitory effect on ALDH3A1, ALDH4A1 or ALDH5A1. Two of the aromatic lactones show selectivity within the ALDH1/2 class, and one appears to be selective for ALDH2 compared to all other isoenzymes tested.
\end{abstract}

\section{Keywords}

aldehyde dehydrogenase; high-throughput screening

\footnotetext{
(C) 2015 Published by Elsevier Ireland Ltd.

*Corresponding Author: Tel: +1 3172782008 thurley@iu.edu (T.D. Hurley).

5 Conflict of Interest

Thomas D. Hurley holds significant financial equity in SAJE Pharma, LLC. However, none of the work described in this study is related to, based on or supported by the company.

Publisher's Disclaimer: This is a PDF file of an unedited manuscript that has been accepted for publication. As a service to our customers we are providing this early version of the manuscript. The manuscript will undergo copyediting, typesetting, and review of the resulting proof before it is published in its final citable form. Please note that during the production process errors may be discovered which could affect the content, and all legal disclaimers that apply to the journal pertain.
} 


\section{Introduction}

Aldehydes are found throughout the body as a product of dietary metabolism and the biotransformation of neurotransmitters, carbohydrates, lipids, and endogenous amino acids [1-3]. In addition numerous aldehydes are present in the environment in smog, motor vehicle exhaust, and formed during the production of plastics $[4,5]$. The accumulation of aldehydes within the body can lead to cytotoxicity and carcinogenesis[3, 4, 6, 7]. The human body has multiple systems of enzymes to alleviate aldehyde stress, one of these being the aldehyde dehydrogenases (ALDHs). The human genome has 19 functional genetic loci for members of the ALDH superfamily, most of which catalyze the NAD $(\mathrm{P})^{+}$-dependent oxidation of aldehydes to their respective carboxylic acids, except for ALDH6A1, which catalyzes the formation of their respective CoA esters[4, 8]. ALDHs are separated into families and subfamilies based on their sequence similarity[9]. The 19 ALDHs share similar but distinct functions within the body due to their varying substrate specificities and gene expression differences. Some gene products, such as ALDH1A1 and ALDH2, are ubiquitously expressed, whereas others are expressed preferentially in certain tissues or during certain periods of development. Naturally occurring mutations within various ALDHs cause human diseases or aversive conditions such as the alcohol flush reaction (ALDH2), Sjogren-Larsson syndrome (ALDH3A2), type II hyperprolinemia (ALDH4A1), and 4-hydroxybutyricaciduria (ALDH5A1) [10-12]. In contrast, members of the ALDH1 and ALDH2 families possess broad and somewhat overlapping substrate specificities making specific assignment of function difficult. Due to the apparent overlap in their function, ALDH-selective chemical probes could aid in gaining a better understanding of the function of these ALDHs, especially those which are within the same family or subfamily.

For several years our lab has been interested in finding novel selective compounds for ALDH1A1, ALDH2, and ALDH3A1[13-15]. ALDH1A1 and ALDH3A1 are cytosolic proteins expressed in many cell types, including ocular tissues where they appear to function as corneal crystallins. Both ALDH1A1 and ALDH3A1 are implicated in providing resistance to certain anti-cancer agents, such as cyclophosphamide[16-18]. ALDH1B1, a mitochondrial enzyme most similar to ALDH2, has recently been shown to be a potential biomarker for colon cancer[19]. ALDH1A1, along with the related cytosolic isoenzymes ALDH1A2 and ALDH1A3, contribute to retinoid metabolism[20]. ALDH1A2 and ALDH1A3 perform crucial functions during embryogenesis, as individual genetic knockout of these two genes in mice are not viable[21,22]. ALDH2 is a mitochondrial enzyme which is most well-known for its role in acetaldehyde metabolism[23]. However, other members of the ALDH family can contribute to acetaldehyde metabolism, especially when ALDH2 activity is reduced by the presence of the ALDH2*2 allele[24]. These other isoenzymes include ALDH1B1 and ALDH1A1[25, 26]. ALDH2, along with ALDH1A1, is implicated in the metabolism of the neurotransmitter dopamine[27]. In addition to these oxidative functions, ALDH2 can contribute to cardiovascular function through its ability to bioactivate nitroglycerin by acting as a nitrate reductase, and has been associated with cardioprotection from ischemic damage by limiting the damage from lipid peroxidation products[28]. Many of the other isoenzymes in the ALDH1, ALDH2, and ALDH3 families are also known to have a cytoprotective role against lipid peroxidation products[1]. The discovery and 
development of isoenzyme-selective inhibitors or activators could prove useful in evaluating the relative contributions that these related ALDH isoenzymes make toward these common substrates.

Here we report the results of a high-throughput screen designed to discover selective modulators of ALDH2 activity. The screen identified 53 compounds from a library of 63000 compounds that modulated the esterase activity of ALDH2. Commercially available compounds were then tested for their effects on the oxidation of aldehyde substrate by ALDH1A1, ALDH1A2, ALDH1A3, ALDH2, ALDH1B1, ALDH3A1, ALDH4A1, and ALDH5A1. This screen discovered a set of four aromatic lactones which exhibit potent inhibition of the ALDH1/2 family members but do not inhibit ALDH3A1, ALDH4A1, or ALDH5A1 activity. Two compounds in particular show selectivity for ALDH2 versus ALDH1A1, and one of those two shows selectivity for ALDH2 versus all other tested isoenzymes. Future characterization will include determining the mechanism by which these aromatic lactones inhibit the ALDH1/2 family of isoenzymes.

\section{Materials and Methods}

\subsection{Materials}

All materials were purchased from Sigma-Aldrich, unless otherwise noted.

\subsection{Expression of ALDH}

ALDH1A1, ALDH1A2, ALDH1A3, ALDH2, ALDH1B1, ALDH3A1, ALDH4A1, and ALDH5A1 were prepared and purified as previously described [13, 15, 29, Morgan et al, Chemico-Biological Interactions (This issue)]

\subsection{High-throughput Screening}

63,000 compounds from ChemDiv were present in $20 \mu \mathrm{L}$ aliquots in $2 \%$ DMSO at $25 \mu \mathrm{M}$ concentration in 384 well plates. Primary screening was completed in 384 well clear bottom plates by measuring the increase in absorbance of para-nitrophenol at $\lambda=405 \mathrm{~nm}$ (molar extinction coefficient of $18,000 \mathrm{M}^{-1} \mathrm{~cm}^{-1}$ in a $50 \mu \mathrm{L}$ assay containing $75 \mathrm{nM}$ ALDH2, 0.8 $\mathrm{mM}$ para-nitrophenylacetate, $10 \mu \mathrm{M}$ library compound, $2 \%$ DMSO, and $25 \mathrm{mM}$ HEPES buffer, pH 7.5 on a Spectromax Plus 384 plate reader for 7 min. Additional wells on each plate either had $100 \mu \mathrm{M}$ daidzin as a control inhibitor or no compound present to determine the normal rate. Compounds which showed greater than $35 \%$ inhibition or $1.3 \mathrm{x}$ activation compared to control reactions were then rescreened for validation. Compounds were cherrypicked from the mother plate to perform the validation screening which used the same esterase assay as the primary screen. Compounds which showed greater than $25 \%$ inhibition or $1.25 \mathrm{x}$ activation in this second screen were chosen for further analysis.

\subsection{ALDH2 Dehydrogenase Screen}

Commercially available compounds validated from the esterase screen were purchased from ChemDiv, ChemBridge, Princeton BioMolecular Research, and InterBioScreen. Compounds were screened for their effect on the oxidation of propionaldehyde by ALDH2. The dehydrogenase activity was assayed spectrophotometrically on a Beckman DU-640 by 
measuring the increase in absorbance of $\mathrm{NAD}(\mathrm{P}) \mathrm{H}$ at $340 \mathrm{~nm}$ (molar extinction coefficient of $6220 \mathrm{M}^{-1} \mathrm{~cm}^{-1}$ ) for 2-3 min. The assay included $200 \mu \mathrm{M} \mathrm{NAD}{ }^{+}, 100 \mu \mathrm{M}$ propionaldehyde, $200 \mathrm{nM}$ ALDH2, $1 \%$ DMSO, and $10 \mu \mathrm{M}$ compound in $25 \mathrm{mM}$ BES, $\mathrm{pH}$ 7.5.

\subsection{Selectivity Assays for Other ALDH Isoenzymes}

Compounds which modulated the oxidation of propionaldehyde by ALDH2 were then tested for their effect on the oxidation of aldehyde substrate by ALDH1A1, ALDH1A2,

ALDH1A3, ALDH1B1, ALDH3A1, ALDH4A1, and ALDH5A1. Compounds were initially tested for selectivity against ALDH1A1 and ALDH3A1. Compounds which were selective against these two isoenzymes were then tested against the other isoenzymes. The assay components for these selectivity assays were designed to provide the maximal stringency toward ALDH2 such that the substrate concentration utilized was $>500$-fold above $\mathrm{K}_{\mathrm{M}}$ for ALDH2 while keeping below 15- fold over $\mathrm{K}_{\mathrm{M}}$ for the other isoenzymes. The dehydrogenase activity was assayed spectrophotometrically on a Beckman DU-640 by measuring the increase in absorbance of $\mathrm{NAD}(\mathrm{P}) \mathrm{H}$ at $340 \mathrm{~nm}$ (molar extinction coefficient of $6220 \mathrm{M}^{-1} \mathrm{~cm}^{-1}$ ) for 2-3 min. For the other ALDH1A family members, the assays included $200 \mu \mathrm{M} \mathrm{NAD}^{+}, 100 \mu \mathrm{M}$ propionaldehyde $\left(\mathrm{K}_{\mathrm{M}}\right.$ values $\sim 50 \mu \mathrm{M}$ for ALDH1A1 and ALDH1A2[30, 31] and $\mathrm{K}_{\mathrm{M}}$ value for ALDH1A3 determined empirically by varying propionaldehyde concentration), 100-200 nM enzyme, $1 \%$ DMSO, and $10 \mu \mathrm{M}$ compound in $25 \mathrm{mM}$ BES, pH 7.5. The assay for ALDH1B1 included $500 \mu \mathrm{M} \mathrm{NAD}^{+}$and $200 \mu \mathrm{M}$ propionaldehyde ( $\left.\mathrm{K}_{\mathrm{M}} \sim 14 \mu \mathrm{M}[32]\right)$. For ALDH4A1, the assay included $1.5 \mathrm{mM} \mathrm{NAD}^{+}$and $20 \mathrm{mM}$ propionaldehyde $\left(\mathrm{K}_{\mathrm{M}} \sim 9 \mathrm{mM}[33]\right)$. For ALDH5A1, the assay included $1.5 \mathrm{mM}$ $\mathrm{NAD}^{+}$and $2 \mathrm{mM}$ propionaldehyde $\left(\mathrm{K}_{\mathrm{M}} \sim 600 \mu \mathrm{M}[34]\right)$. For ALDH3A1, the assay included the commonly utilized substrate benzaldehyde at $300 \mu \mathrm{M}$ ( $\mathrm{K}_{\mathrm{M}} \sim 200 \mu \mathrm{M}$ [35]), $300 \mu \mathrm{M}$ $\mathrm{NADP}^{+}, 20 \mathrm{nM}$ ALDH3A1, 1\% DMSO, and $10 \mu \mathrm{M}$ compound. ALDH3A1, ALDH4A1, and ALDH5A1 also used $100 \mathrm{mM}$ sodium phosphate buffer, $\mathrm{pH}$ 7.5.

\subsection{Substrate Competition Assay}

Steady-state kinetics were used to determine the mode of inhibition by varying inhibitor and substrate concentrations for the prototypical member of this inhibitor family; $2 \mathrm{P} 4$.

Dehydrogenase activity was measured on a Cary Eclipse fluorimeter by monitoring the fluorescence of NADH at $470 \mathrm{~nm}$ after excitation at $320 \mathrm{~nm}$ for 2 min measuring every five seconds. Assay conditions included $5 \mathrm{nM}$ ALDH2, $400 \mu \mathrm{M} \mathrm{NAD}^{+}, 75-600 \mathrm{nM}$ propionaldehyde, $0.2 \%$ DMSO, and 0-80 $\mathrm{nM}$ inhibitor in $100 \mathrm{mM} \mathrm{NaP}_{\mathrm{i}}, \mathrm{pH} 7.0$ in $3 \mathrm{~mL}$ reaction volume. The reaction was initiated by addition of substrate. Due to the low concentrations of substrate used in the assay, all reagents were filtered to remove additional aldehyde contaminants. A standard curve of the fluorescence of NADH from $10 \mathrm{nM}$ to 3000 $\mathrm{nM}$ was used to convert the arbitrary fluorescence units into the amount of NADH produced during the experiment. All data were fit to the tight binding non-linear velocity expressions for competitive, non-competitive, mixed-type non-competitive, and uncompetitive inhibition using SigmaPlot (v12, Enzyme Kinetics Module) to evaluate goodness of fit. LineweaverBurk plots were generated using SigmaPlot to better visualize the mode of inhibition. All data represent the average of four independent experiments of duplicate assays at each concentration. 


\section{Results and Discussion}

\subsection{High-throughput ALDH2 Esterase Screen}

Primary screening identified 1495 compounds from a 63,000 compound library which either activated the esterase activity of ALDH2 at least 1.3 -fold or inhibited it by at least $35 \%$. Of these 1495 compounds, only 57 of them were identified as activators of the esterase activity. In the secondary screen, 53 compounds, 11 activators and 42 inhibitors, modulated the esterase activity of ALDH2 greater than $25 \%$. Figure $1 \mathrm{~A}$ depicts the primary screen results for these 53 hit compounds.

\subsection{ALDH2 Dehydrogenase Screen}

Out of the 53 lead compounds identified by the high-through esterase screen, 45 were commercially available. Compounds were ordered and initially tested for their effect at 10 $\mu \mathrm{M}$ concentration on the oxidation of propionaldehyde by ALDH2 on a Beckman DU-640 (Figure 1B). Several compounds showed no effect on the dehydrogenase activity of ALDH2 at $10 \mu \mathrm{M}$ despite exhibiting effects on the esterase activity in the high-throughput screen. However, eleven compounds did inhibit the oxidation of propionaldehyde by ALDH2 $>20 \%$, and one activated the activity $>20 \%$ (P0146I21).

\subsection{Selectivity of Screening Compounds}

Compounds which modulated the oxidation of propionaldehyde by ALDH $2>20 \%$ were then examined for their effects on the dehydrogenase activity of ALDH1A1 and ALDH3A1 (Table 1). The relative concentrations of substrate used with ALDH1A1 ( 2- to 5-fold over $\left.\mathrm{K}_{\mathrm{M}}\right)$ and ALDH3A1 ( 1.5-fold over $\left.\mathrm{K}_{\mathrm{M}}\right)$ are low compared to the relative concentration of ALDH2 used $\left(\sim 500\right.$-fold $\left.>\mathrm{K}_{\mathrm{M}}\right)$. Therefore, if a compound does modulate the activity of ALDH2 any effect on the other two isoenzymes should be easily measureable. Several of the compounds modulated the oxidation of aldehyde substrate by one or both ALDH1A1 and ALDH3A1. One compound activated the dehydrogenase activity of all three isoenzymes (P00146L21) and a different compound greatly inhibited the activity of all three isoenzymes (P0024N09), both showing a lack of selectivity for any one of the three isoenzymes. Three compounds showed selectivity for ALDH2 at $10 \mu \mathrm{M}$ concentration (P0008L19, P0027H05, and P0038F16). However, a second batch of P0027H05 ordered after initial testing did not inhibit ALDH2 dehydrogenase activity at this concentration. Two other compounds identified by this screen were structurally similar to P0008L19 and P0038F16 (P0038B18 and P0038D18).

\subsection{Characterization of Aromatic Lactones}

The four structurally similar compounds (P0008L19, methyl-2-[(4-methyl-2-oxo-2Hchromen-7-yl)oxy]propanoate, 2BS4; P0038F16, 2,3,5,6-tetramethylfuro[3,2-g]chromen-7one, 2P4; P0038B18, 3,5-dimethyl-6-propylfuro[3,2-g]chromen-7-one, 2CB5; P0038D18, 2,3,5-trimethyl-6-propyl-7H-furo[3,2-g]chromen-7-one, 2P3) are aromatic lactones (Figure $2 \mathrm{~A})$. Three of them (2P3, 2P4, and 2CB5) share the same core three-ringed aromatic structure, differing only in the alkyl chains bound to the aromatic rings. The fourth, 2BS4, shares the two six-membered rings with the other three; however, in place of the furan ring 
2BS4 has a short alkyl chain with a terminal ester group connected to the aromatic system via an ether linkage with the ether oxygen in the same position relative to the oxygen in the furan ring in the other three compounds. 2BS4 also lacks an alkyl chain proximal to the lactone carbonyl.

However, if this class of compounds demonstrates uncompetitive inhibition towards substrate the substrate concentration utilized ( $\sim 500$-fold $>\mathrm{K}_{\mathrm{m}}$ ) for measuring ALDH2 activity would actually enhance the inhibition relative to the other isoenzymes, since the substrate concentrations utilized in their assays were much closer to their respective $\mathrm{K}_{\mathrm{m}}$ values. To exclude this particular scenario, the mode of inhibition with respect to substrate binding for $2 \mathrm{P} 4$ was measured for $\mathrm{ALDH} 2$. The kinetic data for $2 \mathrm{P} 4$ inhibition when varying substrate best fit the tight binding mixed-type non-competitive mode of inhibition (Figure 2B) with a $K_{i}=35 \pm 3 \mathrm{nM}$, propionaldehyde $K_{m}=93 \pm 7 \mathrm{nM}$, and $\mathrm{a}=2.1 \pm 0.3$. This result supports our assay design where the assay for ALDH2 dehydrogenase activity is the most stringent of the isoenzymes with respect to the potential for inhibition.

In addition to the effect of the four aromatic lactones on the dehydrogenase activity of ALDH1A1, ALDH2, and ALDH3A1 previously measured, the lactones were tested for their effect on the oxidation of propionaldehyde by ALDH1A2, ALDH1A3, ALDH1B1, ALDH4A1, and ALDH5A1 at a concentration of $10 \mu \mathrm{M}$ to determine their potency and selectivity for these various isoenzymes (Figure 2C). The substrate concentrations for the additional five isoenzymes used in these assays were between 2- and 14-fold in excess of their respective $\mathrm{K}_{\mathrm{M}}$ for propionaldehyde, considerably less than the $\sim 500$-fold above $\mathrm{K}_{\mathrm{M}}$ for propionaldehyde [36] used for ALDH2, making the ALDH2 inhibition assay the most stringent test compared to the other isoenzymes. Propionaldehyde was used as the common substrate between assays when possible, including the cases of ALDH4A1 and ALDH5A1 where the preferred substrates of $\gamma$-glutamate semialdehyde (ALDH4A1) and succinic semialdehyde (ALDH5A1) were difficult to obtain. In our hands the approximate $\mathrm{K}_{\mathrm{M}}$ value for ALDH1A3 was $\sim 15 \mu \mathrm{M}$.

The compounds can be split into two groups, based on their selectivity and their chemical structures; the first group contains the three tri-cyclic aromatic lactones and the other group contains 2BS4 by itself. None of these compounds inhibit the dehydrogenase activity of ALDH3A1, ALDH4A1, or ALDH5A1 at the concentrations tested. 2CB5, 2P3, and 2P4 inhibit the activity of ALDH2 $>80 \%$ at $10 \mu \mathrm{M}$. These compounds also inhibit the activity of ALDH1A1, ALDH1A2, ALDH1A3, and ALDH1B1, albeit less so than ALDH2. 2P4 does not substantially inhibit ALDH1A1 suggesting the length of the alkyl chain proximal to the carbonyl of the lactone is important for the inhibition of ALDH1A1. A compound with a shorter methyl chain (2P4) does not inhibit ALDH1A1 whereas compounds with longer propyl chains (2CB5 and 2P3) do inhibit ALDH1A1. This finding is consistent with the larger substrate binding channel found in the ALDH1A1 isoenzyme. Additionally, the shorter methyl chain of $2 \mathrm{P} 4$ appears to limit the inhibitory potency of the compound to all of the ALDH1/2 isoenzymes, as $2 \mathrm{P} 4$ shows less inhibition compared to $2 \mathrm{P} 3$ for all of the ALDH1/2 isoenzymes, which may relate to the largely hydrophobic substrate channels found in all of these isoenzymes. Comparing the action of $2 \mathrm{CB} 5$ and $2 \mathrm{P} 3$, the methyl chain proximal to the furan oxygen appears to play a role in the extent of inhibition toward these 
isoenzymes. For each of the ALDH1/2 isozymes 2P3 inhibits greater than 2CB5. However, $2 \mathrm{P} 4$, which shares the methyl chain proximal to the furan oxygen, shows less inhibition than 2CB5 which lacks this particular methyl chain. This highlights the relative importance of the two alkyl chains which differ between these three compounds. It appears that increased hydrophobic interactions from the alkyl chains lead to more effective inhibition, as the compound with the most and longest alkyl chains (2P3) is the only compound of the three to inhibit each ALDH1A and ALDH1B member by at least 60\%. 2BS4 inhibits ALDH2 similar to $2 \mathrm{CB} 5$ and $2 \mathrm{P} 3$, but did not inhibit the activity of any other isoenzyme tested, making it the most selective compound of the four. However, 2BS4 is an ester and may be a competitive substrate for the esterase reaction of ALDH2, which would also compete with aldehyde oxidation since the same active site residues catalyze both reactions. To help determine how 2BS4 may be acting, the effect of 2-[(4-methyl-2-oxo-2H-chromen-7yl)oxy]propanoic acid (the free acid form of 2BS4) and 4-methyl-7-[(3-oxobutan-2yl)oxy]-2H-chromen-2-one (the methyl ketone of 2BS4) on the oxidation of propionaldehyde by ALDH2 was examined. Neither the free acid nor the terminal ketone analogs inhibited ALDH2, suggesting that the ester group itself, and not the carbonyl, is important for the inhibition of ALDH2. The lack of inhibition by the free acid is consistent with the fact that acid products are generally not inhibitory for most ALDH2/substrate combinations. In addition, pre-incubation of 2BS4 with ALDH2 for up to sixty minutes showed no evidence of a time-dependent mechanism of inhibition and no time-dependent change to the apparent $\mathrm{IC}_{50}$ for 2BS4 inhibition of ALDH2 (data not shown), suggesting that if hydrolysis is occurring it is slow relative to para-nitrophenylacetate hydrolysis or propionaldehyde oxidation.

\section{Conclusion}

The aromatic lactones identified by this screen are potent $\left(2 \mathrm{P} 4 \mathrm{~K}_{\mathrm{i}} \approx 35 \mathrm{nM}\right.$ for ALDH2) inhibitors of the ALDH1/2 isoenzymes. These compounds, upon further development, could be used to better determine the functions of each isoenzyme. These compounds also have therapeutic potential as inhibitors of the ALDH1/2 family of enzymes have medicinal applications as modulators of dopamine metabolism, antidipsotropic drugs, and as chemotherapy sensitizers. However, a strictly ALDH2-selective compound remains elusive as separating the inhibition of ALDH2 from the inhibition of ALDH1A1 has been difficult. Additionally, the effect of many currently used ALDH1A1 and ALDH2 inhibitors (for instance, disulfiram, daidzin or DEAB) on the related but lesser-studied ALDH1A2, ALDH1A3, and ALDH1B1 has not been fully examined. Two of the lactones reported here, 2P4 and 2BS4, appear to inhibit ALDH2 without inhibiting ALDH1A1 achieving the separation in inhibition rarely seen in previous compounds. Future studies will include determining the dose-dependent effects of these four compounds on the various isoenzymes and determining the binding site of these compounds through $\mathrm{x}$-ray crystallography to further the development of these aromatic lactones as ALDH1/2 inhibitors, with the hope that slight structural modifications will yield an ALDH1A1-selective or ALDH2-selective inhibitor. 


\section{Acknowledgments}

The authors would like to thank Cindy Morgan, Bibek Parajuli, and especially Lan Min Zhai for help with the production and purification of the various ALDH isozymes. The authors would also like to thank Lan Chen, PhD, and the Indiana University Chemical Genomics Core for providing access to their facilities to complete the highthroughput screening and the chemical libraries and John Turchi, PhD, for use of the Cary Eclipse fluorimeter. This research was supported by NIH R01-AA018123. Cameron Buchman was supported by R13-AA023149 to present this work at the $17^{\text {th }}$ Enzymology and Molecular Biology of Carbonyl Metabolism meeting held in Skytop, PA, USA.

\section{References}

1. Vasiliou V, Pappa A, Estey T. Role of human aldehyde dehydrogenases in endobiotic and xenobiotic metabolism. Drug metabolism reviews. 2004; 36:279-299. [PubMed: 15237855]

2. Vasiliou V, Pappa A, Petersen DR. Role of aldehyde dehydrogenases in endogenous and xenobiotic metabolism. Chemico-biological interactions. 2000; 129:1-19. [PubMed: 11154732]

3. O'Brien PJ, Siraki AG, Shangari N. Aldehyde sources, metabolism, molecular toxicity mechanisms, and possible effects on human health. Critical reviews in toxicology. 2005; 35:609-662. [PubMed: 16417045]

4. Marchitti SA, Brocker C, Stagos D, Vasiliou V. Non-P450 aldehyde oxidizing enzymes: the aldehyde dehydrogenase superfamily. Expert opinion on drug metabolism \& toxicology. 2008; 4:697-720. [PubMed: 18611112]

5. Wong RH, Wang JD, Hsieh LL, Du CL, Cheng TJ. Effects on sister chromatid exchange frequency of aldehyde dehydrogenase 2 genotype and smoking in vinyl chloride workers. Mutation research. 1998; 420:99-107. [PubMed: 9838066]

6. Cheng G, Shi Y, Sturla SJ, Jalas JR, McIntee EJ, Villalta PW, Wang M, Hecht SS. Reactions of formaldehyde plus acetaldehyde with deoxyguanosine and DNA: formation of cyclic deoxyguanosine adducts and formaldehyde cross-links. Chemical research in toxicology. 2003; 16:145-152. [PubMed: 12588185]

7. Cho YJ, Wang H, Kozekov ID, Kurtz AJ, Jacob J, Voehler M, Smith J, Harris TM, Lloyd RS, Rizzo CJ, Stone MP. Stereospecific formation of interstrand carbinolamine DNA cross-links by crotonaldehyde- and acetaldehyde-derived alpha-CH3-gamma-OH-1,N2-propano-2'deoxyguanosine adducts in the $5^{\prime}-\mathrm{CpG}-3^{\prime}$ sequence. Chemical research in toxicology. 2006; 19:195-208. [PubMed: 16485895]

8. Kedishvili NY, Popov KM, Rougraff PM, Zhao Y, Crabb DW, Harris RA. CoA-dependent methylmalonate-semialdehyde dehydrogenase, a unique member of the aldehyde dehydrogenase superfamily. cDNA cloning, evolutionary relationships, and tissue distribution. The Journal of biological chemistry. 1992; 267:19724-19729. [PubMed: 1527093]

9. Vasiliou V, Bairoch A, Tipton KF, Nebert DW. Eukaryotic aldehyde dehydrogenase (ALDH) genes: human polymorphisms, and recommended nomenclature based on divergent evolution and chromosomal mapping. Pharmacogenetics. 1999; 9:421-434. [PubMed: 10780262]

10. Rizzo WB, Carney G. Sjogren-Larsson syndrome: diversity of mutations and polymorphisms in the fatty aldehyde dehydrogenase gene (ALDH3A2). Human mutation. 2005; 26:1-10. [PubMed: 15931689]

11. Vasiliou V, Pappa A. Polymorphisms of human aldehyde dehydrogenases. Consequences for drug metabolism and disease. Pharmacology. 2000; 61:192-198. [PubMed: 10971205]

12. Geraghty MT, Vaughn D, Nicholson AJ, Lin WW, Jimenez-Sanchez G, Obie C, Flynn MP, Valle D, Hu CA. Mutations in the Delta1-pyrroline 5-carboxylate dehydrogenase gene cause type II hyperprolinemia. Human molecular genetics. 1998; 7:1411-1415. [PubMed: 9700195]

13. Parajuli B, Kimble-Hill AC, Khanna M, Ivanova Y, Meroueh S, Hurley TD. Discovery of novel regulators of aldehyde dehydrogenase isoenzymes. Chemico-biological interactions. 2011; 191:153-158. [PubMed: 21349255]

14. Kimble-Hill AC, Parajuli B, Chen CH, Mochly-Rosen D, Hurley TD. Development of selective inhibitors for aldehyde dehydrogenases based on substituted indole-2,3-diones. Journal of medicinal chemistry. 2014; 57:714-722. [PubMed: 24444054] 
15. Parajuli B, Georgiadis TM, Fishel ML, Hurley TD. Development of selective inhibitors for human aldehyde dehydrogenase 3A1 (ALDH3A1) for the enhancement of cyclophosphamide cytotoxicity. Chembiochem : a European journal of chemical biology. 2014; 15:701-712. [PubMed: 24677340]

16. Hilton J. Role of aldehyde dehydrogenase in cyclophosphamide-resistant L1210 leukemia. Cancer research. 1984; 44:5156-5160. [PubMed: 6488175]

17. Sreerama L, Sladek NE. Identification and characterization of a novel class 3 aldehyde dehydrogenase overexpressed in a human breast adenocarcinoma cell line exhibiting oxazaphosphorine-specific acquired resistance. Biochemical pharmacology. 1993; 45:2487-2505. [PubMed: 8328987]

18. Moreb JS, Mohuczy D, Ostmark B, Zucali JR. RNAi-mediated knockdown of aldehyde dehydrogenase class- $1 \mathrm{~A} 1$ and class-3A1 is specific and reveals that each contributes equally to the resistance against 4-hydroperoxycyclophosphamide. Cancer chemotherapy and pharmacology. 2007; 59:127-136. [PubMed: 16614850]

19. Chen Y, Orlicky DJ, Matsumoto A, Singh S, Thompson DC, Vasiliou V. Aldehyde dehydrogenase 1B1 (ALDH1B1) is a potential biomarker for human colon cancer. Biochemical and biophysical research communications. 2011; 405:173-179. [PubMed: 21216231]

20. Duester G. Families of retinoid dehydrogenases regulating vitamin A function: production of visual pigment and retinoic acid. European journal of biochemistry / FEBS. 2000; 267:4315-4324. [PubMed: 10880953]

21. Niederreither K, Subbarayan V, Dolle P, Chambon P. Embryonic retinoic acid synthesis is essential for early mouse post-implantation development. Nature genetics. 1999; 21:444-448. [PubMed: 10192400]

22. Dupe V, Matt N, Garnier JM, Chambon P, Mark M, Ghyselinck NB. A newborn lethal defect due to inactivation of retinaldehyde dehydrogenase type 3 is prevented by maternal retinoic acid treatment. Proceedings of the National Academy of Sciences of the United States of America. 2003; 100:14036-14041. [PubMed: 14623956]

23. Klyosov AA, Rashkovetsky LG, Tahir MK, Keung WM. Possible role of liver cytosolic and mitochondrial aldehyde dehydrogenases in acetaldehyde metabolism. Biochemistry. 1996; 35:4445-4456. [PubMed: 8605194]

24. Farres J, Wang X, Takahashi K, Cunningham SJ, Wang TT, Weiner H. Effects of changing glutamate 487 to lysine in rat and human liver mitochondrial aldehyde dehydrogenase. A model to study human (Oriental type) class 2 aldehyde dehydrogenase. The Journal of biological chemistry. 1994; 269:13854-13860. [PubMed: 7910607]

25. Ueshima Y, Matsuda Y, Tsutsumi M, Takada A. Role of the aldehyde dehydrogenase-1 isozyme in the metabolism of acetaldehyde. Alcohol and alcoholism. 1993; 1B:15-19. [PubMed: 8003124]

26. Stewart MJ, Malek K, Xiao Q, Dipple KM, Crabb DW. The novel aldehyde dehydrogenase gene, ALDH5, encodes an active aldehyde dehydrogenase enzyme. Biochemical and biophysical research communications. 1995; 211:144-151. [PubMed: 7779080]

27. Marchitti SA, Deitrich RA, Vasiliou V. Neurotoxicity and metabolism of the catecholaminederived 3,4-dihydroxyphenylacetaldehyde and 3,4-dihydroxyphenylglycolaldehyde: the role of aldehyde dehydrogenase. Pharmacological reviews. 2007; 59:125-150. [PubMed: 17379813]

28. Mayer B, Beretta M. The enigma of nitroglycerin bioactivation and nitrate tolerance: news, views and troubles. British journal of pharmacology. 2008; 155:170-184. [PubMed: 18574453]

29. Hammen PK, Allali-Hassani A, Hallenga K, Hurley TD, Weiner H. Multiple conformations of NAD and NADH when bound to human cytosolic and mitochondrial aldehyde dehydrogenase. Biochemistry. 2002; 41:7156-7168. [PubMed: 12033950]

30. Xiao T, Zhang M, Ansari N. Studies on aldehyde dehydrogenase 1(ALDH1A1), a crucial enzyme in maintaining the lens clarity under oxidative stress. Invest Ophth Vis Sci. 2005; 46

31. Wang X, Penzes P, Napoli JL. Cloning of a cDNA encoding an aldehyde dehydrogenase and its expression in Escherichia coli. Recognition of retinal as substrate. The Journal of biological chemistry. 1996; 271:16288-16293. [PubMed: 8663198]

32. Stagos D, Chen Y, Brocker C, Donald E, Jackson BC, Orlicky DJ, Thompson DC, Vasiliou V. Aldehyde dehydrogenase 1B1: molecular cloning and characterization of a novel mitochondrial 
acetaldehyde-metabolizing enzyme. Drug metabolism and disposition: the biological fate of chemicals. 2010; 38:1679-1687. [PubMed: 20616185]

33. Forte-McRobbie CM, Pietruszko R. Purification and characterization of human liver "high Km" aldehyde dehydrogenase and its identification as glutamic gamma-semialdehyde dehydrogenase. The Journal of biological chemistry. 1986; 261:2154-2163. [PubMed: 3944130]

34. Ryzlak MT, Pietruszko R. Human brain glyceraldehyde-3-phosphate dehydrogenase, succinic semialdehyde dehydrogenase and aldehyde dehydrogenase isozymes: substrate specificity and sensitivity to disulfiram. Alcoholism, clinical and experimental research. 1989; 13:755-761.

35. Pappa A, Estey T, Manzer R, Brown D, Vasiliou V. Human aldehyde dehydrogenase 3A1 (ALDH3A1): biochemical characterization and immunohistochemical localization in the cornea. The Biochemical journal. 2003; 376:615-623. [PubMed: 12943535]

36. Klyosov AA. Kinetics and specificity of human liver aldehyde dehydrogenases toward aliphatic, aromatic, and fused polycyclic aldehydes. Biochemistry. 1996; 35:4457-4467. [PubMed: 8605195] 


\section{Highlights}

- High-thoughput screening identified a novel class of ALDH1/2-directed inhibitors.

- This class shares an aromatic lactone as a structural feature.

- Two lactones appear to be selective for ALDH2 versus ALDH1A1. 

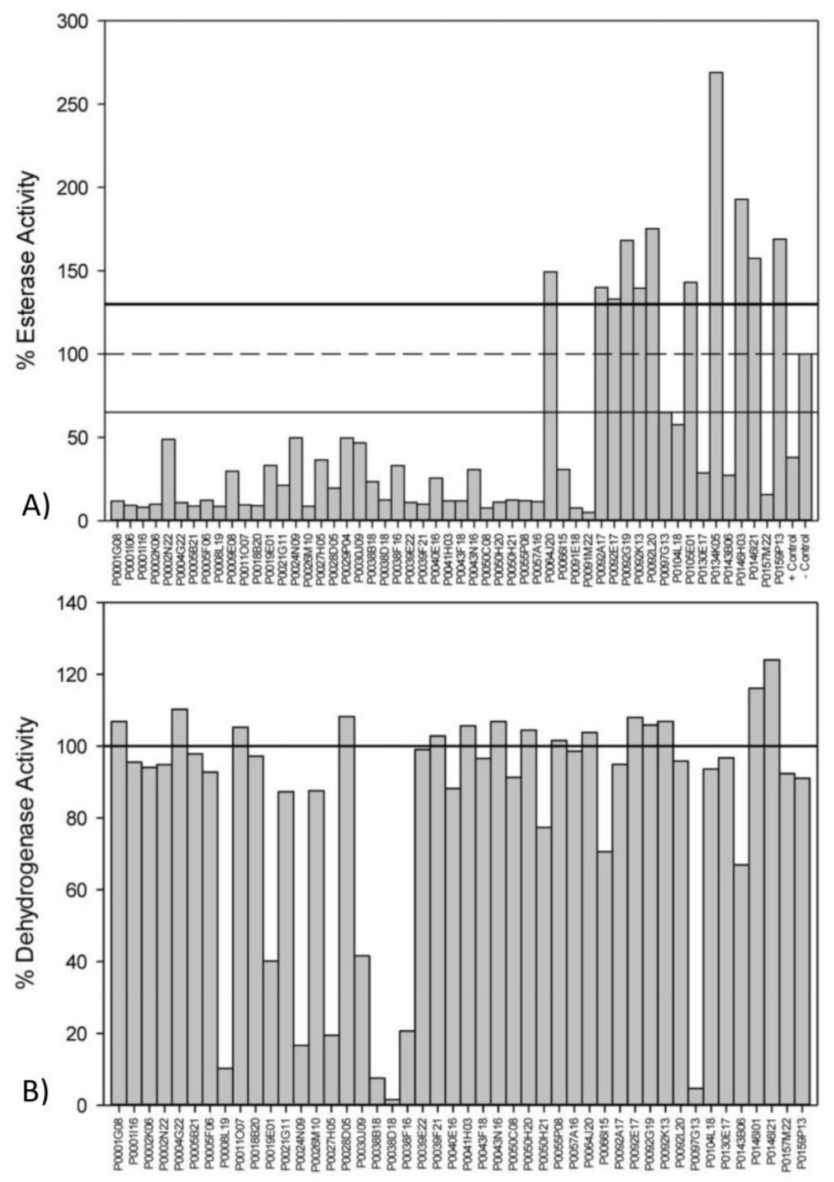

Figure 1.

A) Primary screen results for the 53 lead compounds. The esterase activity of ALDH2 with $10 \mu \mathrm{M}$ compound present was compared to the activity of ALDH2 in the absence of compound (- control). Selection criteria was either $>130 \%$ activity or $<65 \%$ activity. The positive control was $100 \mu \mathrm{M}$ daidzin. B) Effect of the 53 lead compounds on the dehydrogenase activity of ALDH2. The oxidation of propionaldehyde by ALDH2 with 10 $\mu \mathrm{M}$ compound present was compared to the activity of ALDH2 in the absence of compound. Value is an average of 2 trials. 

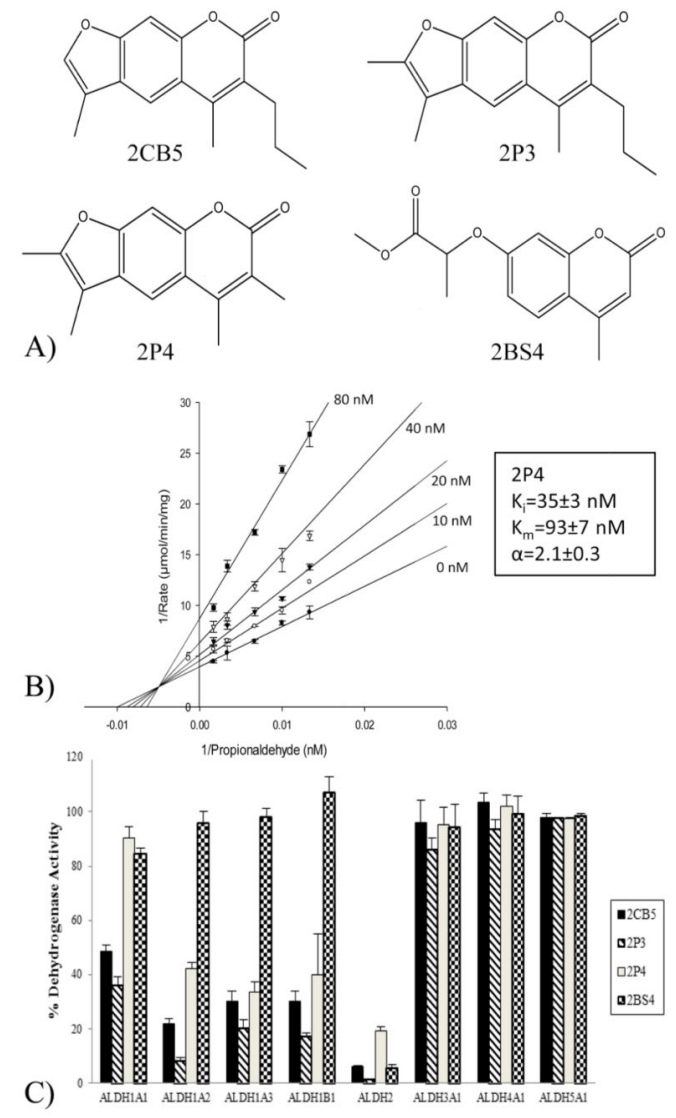

Figure 2.

A) Structures of the aromatic lactones. B) Lineweaver-Burk representation of the fit to the tight binding mixed-type non-competitive inhibition equation for $2 \mathrm{P} 4$ versus varying propionaldehyde concentrations with ALDH2. $\mathrm{K}_{\mathrm{i}}, \mathrm{K}_{\mathrm{m}}$, and a values are presented with standard error. The plot is a representative single experiment from one of four independent experiments. C) \% Dehydrogenase activity for ALDH2, ALDH1B1, ALDH1A1,

ALDH1A2, ALDH1A3, ALDH3A1, ALDH4A1, and ALDH5A1 in the presence of $10 \mu \mathrm{M}$ compound. Value is the average of at least three independent trials $(n \geq 3)$ with standard error, except for the measurement of ALDH1B1 activity with 2P4 (n=2). 


\section{Table 1}

Initial selectivity results for the most selective compounds. Listed is the dehydrogenase activity of ALDH2, ALDH1A1, and ALDH3A1 in the presence of $10 \mu \mathrm{M}$ compound as the percent activity compared to the reaction without compound present.

\begin{tabular}{|cccc|}
\hline Compound & ALDH2 & ALDH1A1 $^{*}$ & ALDH3A1 \\
\hline P0038D18 & 1.5 & 40.8 & 77.2 \\
\hline P0097G13 & 4.7 & 73.5 & 61.3 \\
\hline P0038B18 & 7.6 & 58.6 & 87.6 \\
\hline P0008L19 & 10.3 & 82.8 & 94.5 \\
\hline P0024N09 & 16.6 & 6.1 & 6.1 \\
\hline P0027H05 & 19.5 & 88.0 & 82.4 \\
\hline P0038F16 & 20.7 & 89.3 & 87.8 \\
\hline P0019E01 & 40.1 & 158.9 & 85 \\
\hline P0030J09 & 41.6 & 69.7 & 77.3 \\
\hline P0143B06 & 66.9 & 29.1 & 45.4 \\
\hline P0066I15 & 70.6 & 2.0 & N/M \\
\hline P0146I21 & 124 & 126.9 & 119.7 \\
\hline$*$ & & \\
N121S polymorphism & & \\
N/M = Not Measured & &
\end{tabular}

